\title{
NLFK4ALL: AN OPEN-SOURCE DEMONSTRATION TOOLBOX FOR COMPUTATIONALLY EFFICIENT NONLINEAR FROUDE-KRYLOV FORCE CALCULATIONS
}

\author{
GIUSEPPE GIORGI ${ }^{\dagger, 1, *}$, GIOVANNI BRACCO ${ }^{\dagger, 2}$ AND GIULIANA MATTIAZZO $^{\dagger, 3}$ \\ ${ }^{1}$ giuseppe.giorgi@ polito.it \\ 2 giovanni.bracco@polito.it \\ 3 giuliana.mattiazzo@polito.it \\ * corresponding author \\ ${ }^{\dagger}$ Department of Mechanical and Aerospace Engineering, Politecnico di Torino, Turin, Italy
}

Key words: Wave-structure interaction, Nonlinear Froude-Krylov force, Nonlinear hydrodynamic modelling, Floating axisymmetric buoys, NLFK4ALL

\begin{abstract}
Accurate modelling of wave-structure interaction is essential for a successful and reliable design of offshore structures and their ancillary systems. However, the fidelity of mathematical models is challenged when nonlinearities become significant, i.e. when the floater operates in severe sea states and/or it shows large dynamic responses compared to the incoming wave. This is normally the case for wave energy converters (WECs) because large motions are desirable for better power extraction, while conventional offshore structures are usually designed to prevent large responses and ensure stability. Fast computation is a further mandatory requirement for mathematical models that are used for design purposes. This is particularly true for wave energy applications, since extensive parametric studies are needed to minimize the cost of energy, i.e. increase power extraction capabilities while limiting capital and operational expenditures. Furthermore, model-based control strategies, essential for substantial increase of the WEC performance, require representative mathematical models. The requirements of accuracy and low computational time are usually contrasting, so an appropriate compromise should be defined. This paper proposes a nonlinear Froude-Krylov (NLFK) force model for axisymmetric floaters. The symmetry of the geometry (common for WECs) is exploited to achieve a low computational time (about real-time computation). Furthermore, since NLFK forces are the main nonlinearities for such devices, the obtained accuracy is higher than linear models. An open-source Matlab demonstration toolbox is introduced, called NLFK4ALL (doi: 10.5281/zenodo.3544848), which provides a ready-to-use implementation of the NLFK approach in six degrees of freedom. Accuracy and computational aspects of the method are here discussed, such as the complexity of the analytical description of the intersection between free surface elevation and the floater, and the impact of tolerances on the convergence of the numerical integration.
\end{abstract}




\section{INTRODUCTION}

This paper purports to present an open-source demonstration toolbox, called NLFK4ALL [1], which aims at providing a ready-to-use implementation of a computationally efficient integration method of surface integrals over axisymmetric bodies. The content of the paper and toolbox are developed in the context of nonlinear hydrodynamic modelling for wave energy converters. Therefore, most of the discussion and considerations are related to the challenges and requirements of this specific area of ocean engineering. However, the tools herein provided have general validity and broader applicability. In fact, such a mathematical framework allows to displace an axisymmetric geometry in 6 degrees of freedom (DoFs) and to compute arbitrary surface integrals on a time-varying portion of the body. In the specific application case of the computation of NLFK forces, the integrand is the undisturbed pressure field, but any other function could be integrated using the same mathematical framework.

The rationale behind the development of this methodology is the critical need for a better compromise of accuracy and computational time with respect to state-of-the-art mathematical models in wave energy conversion applications. Economic viability is the sine qua non condition for commercial competitiveness and industrial sustainability of the wave energy conversion sector and it can be achieved only through substantial reduction of costs and increase in performance. Both such objectives can be accomplished only through holistic design, control, and optimization studies, the effectiveness of which strongly depends on the accuracy of mathematical models $[2,3]$. However, a major limiting factor for the feasibility of application of a particular mathematical model is the computational time.

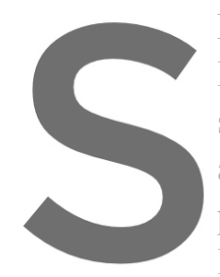
Exploring the computation/fidelity continum [4], th
low computational cost. Considering the power-prod
survivability conditions, partially-nonlinear potenti
appropriate trade-of between conputational time and
point absorbers (device relatively small compared Krylov forces, while radia on and diffraction effor
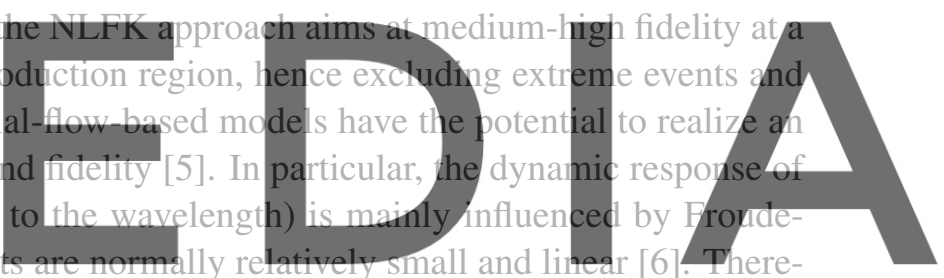

fore, NLFK approaches for point absorbers are particularly accurate, where the undisturbed pressure

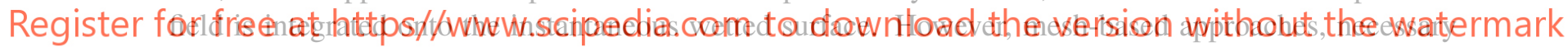

for geometry of arbitrary complexity, are renown to be slow, due to time-consuming re-meshing of the submerged surface [7, 8]. If the floater is assumed to be axisymmetric (not a restrictive assumptions for point absorbers, which are normally axisymmetric), a computationally efficient NLFK formulation exists, relying on the analytical representation of the wetted surface, hence needless of a mesh. If rotations are negligible, algebraic solutions can be obtained [9], otherwise numerical integration is required [10]. Although a fair comparison is challenging, due to different coding languages (Fortran versus Matlab), such numerical solution of the analytically defined NLFK forces for axisymmetric devices is between one and two orders of magnitude faster than a mesh-based approach [11]. Although geometry of arbitrary complexity still require a mesh-based approach, such as $[12,13]$, the methodology presented in this paper can be expanded to prismatic floaters, such as $[14,15]$, since the three-dimensional problem can be reduced to a two-dimensional problem in the same way as for axisymmetric floaters.

The Matlab demonstration toolbox has the objective of providing the user with a set of scripts that can quickly and pragmatically show the capability of this method. The code is completely open-source, modifiable and reusable. This paper, after briefly presenting the theoretical background and computational performance of the method, details the numerical identification of the intersection between the free 

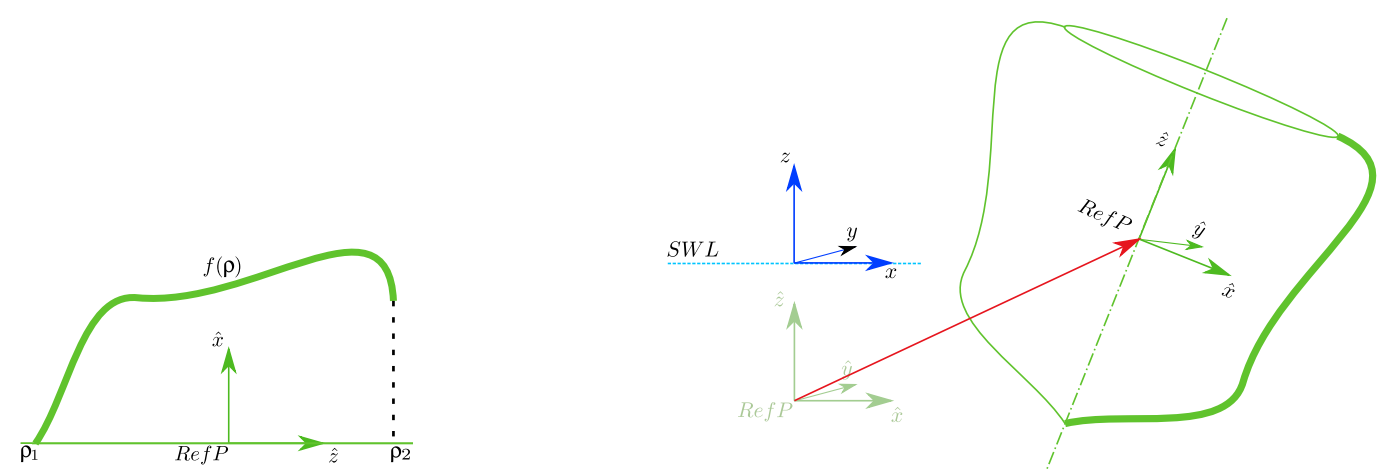

Figure 1: Generic profile of revolution in the BF (left); relative position between frames after displacement (right).

surface elevation and the buoy, and provides a critical discussion of the performance of the numerical integration scheme and the impact of the choice of its absolute and relative tolerances.

\section{Mathematical framework and nonlinear Froude-Krylov force computation}

In this section, the general theoretical framework of the NLFK force integration is presented. Section 2.1 presents the two frames of reference, one world-fixed and one body-fixed, and the mapping function from one frame to the other. Section 2.2 presents three different strategies to compute the upper bound of

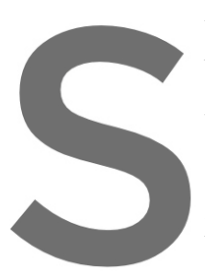
the instantaneous wetted

buoy. Although this math the instantaneous surface,

2.1 World and body-fixed frames
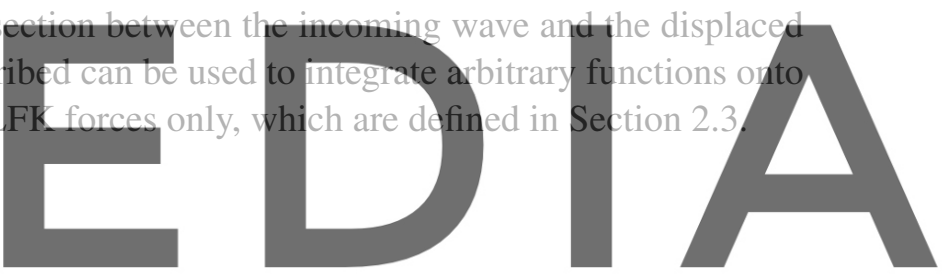

Two right-handed frames of reference are used hereafter:

Register for frepoft.ftafpsh (wwww.scipedia.com to download the version without the watermark

- Body-fixed frame (BF): $(\hat{x}, \hat{y}, \hat{z})^{\prime}$

A given position point $\mathbf{p}$ in the three-dimensional (3D) space can be expressed (decomposed) in either the WF or the BF, and denoted by the notation $\mathbf{p}_{\mathrm{WF}}$ or $\mathbf{p}_{\mathrm{BF}}$, respectively. The world-frame of reference $(x, y, z)^{\prime}$ is inertial, with the origin at the still water level (SWL), the $x$-axis positive in the direction of propagation of the wave, and the $z$-axis positive upwards. The body-fixed frame of reference $(\hat{x}, \hat{y}, \hat{z})^{\prime}$ is not inertial, with the origin fixed at a reference point (RefP), usually either at the centre of gravity $(\mathrm{CoG})$ or at the SWL, with the $z$-axis pointing upwards along the axis of the axisymmetric body.

The lateral surface of a generic axisymmetric geometry can be described by means of cylindrical coordinates. Note that the whole wetted surface of a floating axisymmetric buoy may also need discs, which are more conveniently represented with polar coordinates. Using cylindrical coordinates, the axisymmetric geometry is defined by the revolution of a generic function $f(\rho)$, between $\rho_{1}$ and $\rho_{2}$, as shown at the left side of Figure 1. Note that $f(\rho)$ must be defined so that the origin of the BF (i.e. the RefP) is at $\hat{z}=\rho=0$. Therefore, the parametrization of the axisymmetric geometry, in the BF, is shown in equation (1). Note that it is also possible to consider only a portion of the axisymmetric body by defining appropriate $\vartheta_{1}$ 
and $\vartheta_{2}$, instead of the full revolution from $-\pi$ to $\pi$.

$$
\left\{\begin{array}{l}
\hat{x}(\rho, \vartheta)=f(\rho) \cos \vartheta \\
\hat{y}(\rho, \vartheta)=f(\rho) \sin \vartheta \quad, \quad \vartheta \in[-\pi, \pi) \wedge \rho \in\left[\rho_{1}, \rho_{2}\right] \\
\hat{z}(\rho, \vartheta)=\rho
\end{array}\right.
$$

The displacement of the RefP in WF coordinates is denoted by $\left(x_{R}, y_{R}, z_{R}\right)_{\mathrm{WF}}^{\prime}$. The right side of Figure 1 shows the body after a displacement $\left(x_{R}, y_{R}, z_{R}\right)_{W F}^{\prime}$ and a rotation $(\phi, \theta, \psi)^{\prime}$. Note that such rotations are considered about the three body-fixed axes and about the RefP. The 3-2-1 Euler rotation sequence is applied, commonplace in ocean engineering $[16,17]$, with $\phi$ being the roll angle around the $\hat{x}$-axis, $\theta$ the pitch angle around the $\hat{y}$-axis, and $\psi$ the yaw angle around the $\hat{z}$-axis. The $3-2-1$ rotation matrix $\mathcal{R}$ is defined as follows:

$$
R=\left[\begin{array}{ccc}
c \psi & -s \psi & 0 \\
s \psi & c \psi & 0 \\
0 & 0 & 1
\end{array}\right]\left[\begin{array}{ccc}
c \theta & 0 & s \theta \\
0 & 1 & 0 \\
-s \theta & 0 & c \theta
\end{array}\right]\left[\begin{array}{ccc}
1 & 0 & 0 \\
0 & c \phi & -s \phi \\
0 & s \phi & c \phi
\end{array}\right]
$$

Where $c$ and $s$ stand for $\cos ()$ and $\sin ()$, respectively. Since rotations are defined around the RefP and about the body-fixed axes, the rotation matrix $R$ in (2) pre-multiplies the BF-position vector $(\hat{x}, \hat{y}, \hat{z})^{\prime}$, applying a rotation around the origin of the BF, which is also the RefP of the body. After the rotation,

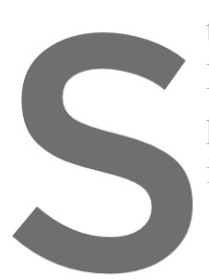
the axes of the two frapes

Note that $C$ is the position position of the two frantes is more convenient to expand the
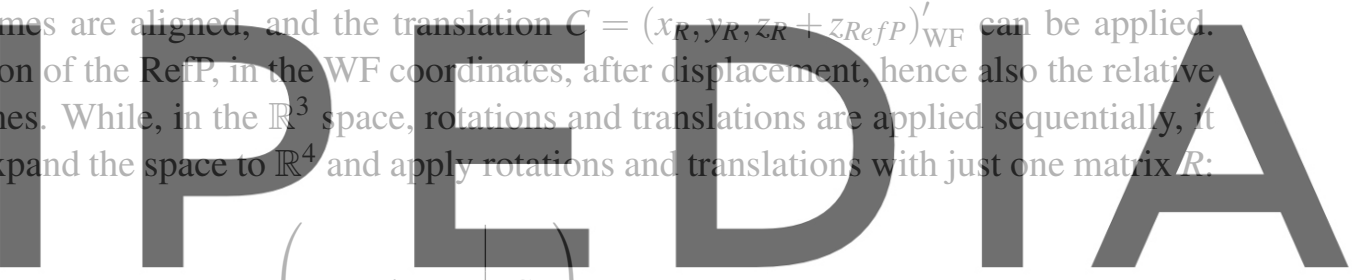

Register for free at https//www.scipedia.com to download the version without the watermark

\subsection{Intersection}

The main advantage of the approach proposed in this paper is the ability to analytically represent the instantaneous wetted surface, which implies the analytical definition of the intersection between the body-surface and an arbitrary function for the free surface elevation ( $\eta$ ). Since, as discussed in Sect. 2.3, it is convenient to define the integrals in the BF, a mapping of the free surface elevation from the WF onto the $B F(\hat{\eta})$ is required. Finding such an intersection translates into finding $\rho=\hat{\eta}(\vartheta)$, which is a parametric representation of the free surface in the BF.

The 4D definition, in the WF, of the wave surface is $\Gamma=(x, y, \eta(x), 1)^{\prime}$. Consequently, $\Gamma$ in the BF becomes: $\hat{\Gamma}=R^{-1} \Gamma$. The intersection is found by equating $\rho$ to the third line of $\hat{\Gamma}$, i.e. $\rho=(0,0,1,0) R^{-1} \Gamma$. However, $\Gamma$ must be expressed as a function of parametric variables. It follows that the intersection is the solution of the following nonlinear equation: 

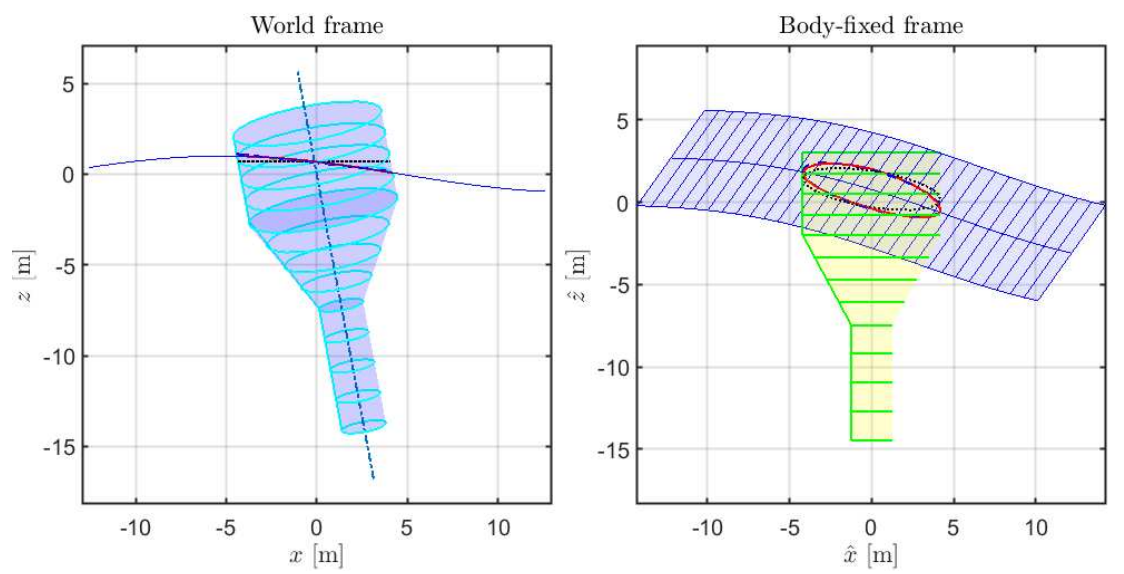

Figure 2: World and body-fixed frames for a Corpower-like device after an arbitrary 6-DoF displacement, with a steep wave of $5 \mathrm{~s}$ period and $2 \mathrm{~m}$ height (ratio between floater diameter and wavelength is about $10 \%$ ). The intersection with the free surface is shown using the constant approximation $(\cdot-\cdot)$, linear approximation $(--=)$, and exact solution $(-)$.
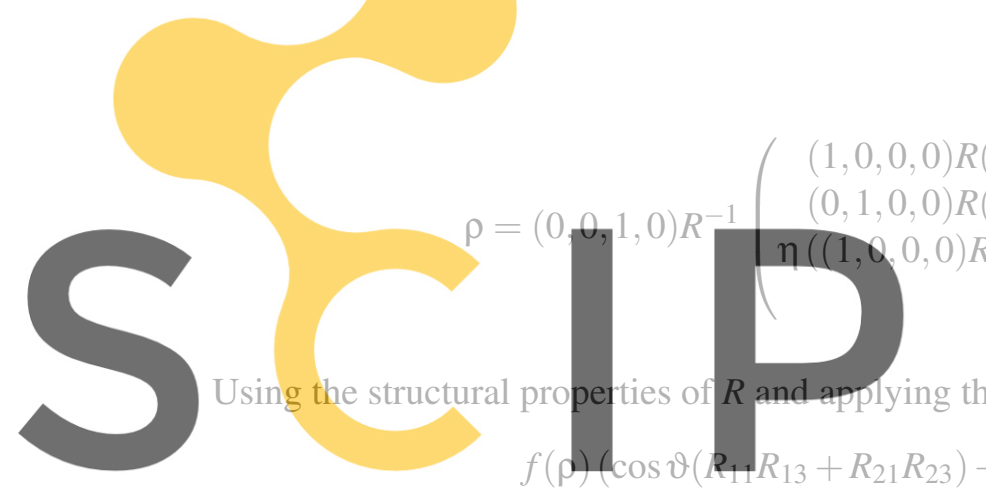

$(1,0,0,0) R(f(\rho) \cos \vartheta, f(\rho) \sin \vartheta, \rho, 1)^{\prime}$ $(0,1,0,0) R(f(\rho) \cos \vartheta, f(\rho) \sin \vartheta, \rho, 1)^{\prime}$
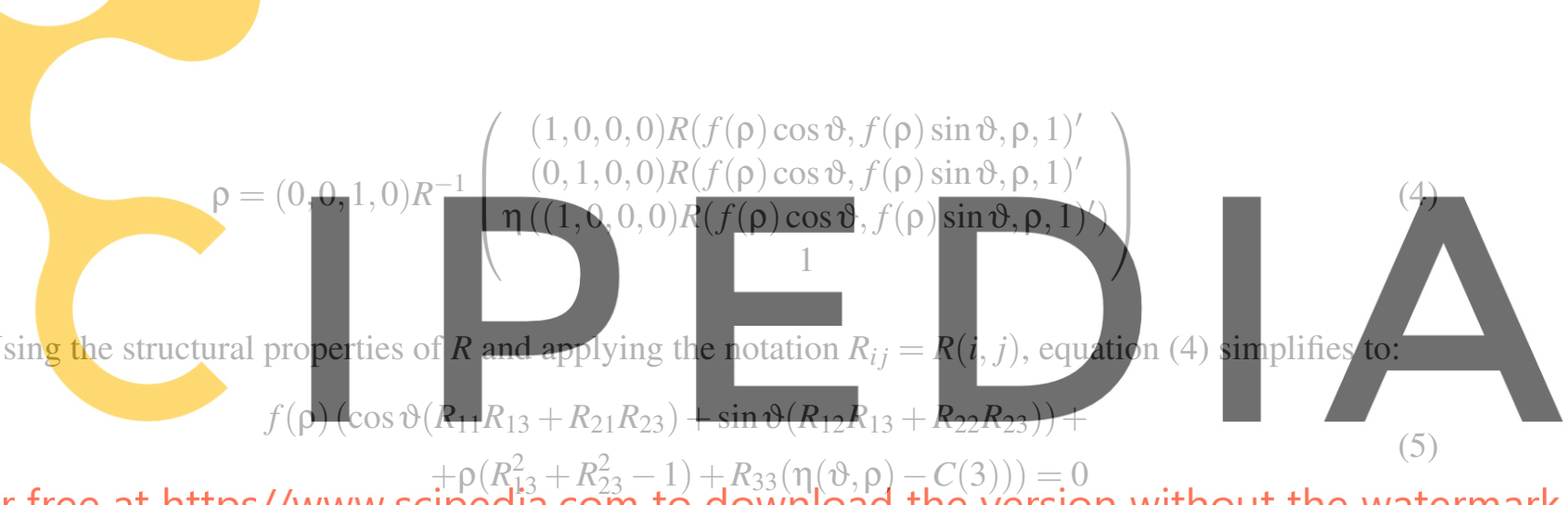

(5)

\section{Register for free at https//www.scipedia.com to download the version without the watermark}

The zeros of equation (4) represent the exact solution of the intersection between the body and the

free surface elevation, but a numerical solution to such a nonlinear problem is required. However, it is reasonable to assume the free surface elevation to be either locally constant, or with a linear dependence on $x$. In fact, in normal applications, the wave steepness is relatively low, and the wavelength is much longer than the characteristic dimensions of the geometry. If $\eta$ is assumed constant (horizontal), $\eta\left(x_{R}\right)$ can be used as a reasonable approximation, accurate if the wavelength is much longer than the diameter of the floater. Alternatively, a linear regression can be considered instead. Selecting an approximate range of $x$ where the buoy is located, a polynomial fit can be performed, so that $\eta \simeq p_{0} x+p_{1}$. The solution for a constant $\eta$ is obtained for $p_{0}=0$ and $p_{1}=\eta\left(x_{R}\right)$.

In order to algebraically compute an approximated intersection, it is also assumed that $f(\rho)$ is either constant or linear, resulting in a cylinder or a cone, respectively. Hereafter, the more general solution for a cone is given, with $f(\rho)=m \rho+q$, which simplifies to a cylinder when $m=0$. A solution with other $f(\rho)$ may be achieved, but they are not common application cases, since the vast majority of buoys, if not the totality, is composed of cones and cylinders. Therefore, solving equation (5) with $\eta \simeq\left(p_{0} x+p_{1}\right)$ and $f(\rho)=(m \rho+q)$, it is found that the intersection is given by: 

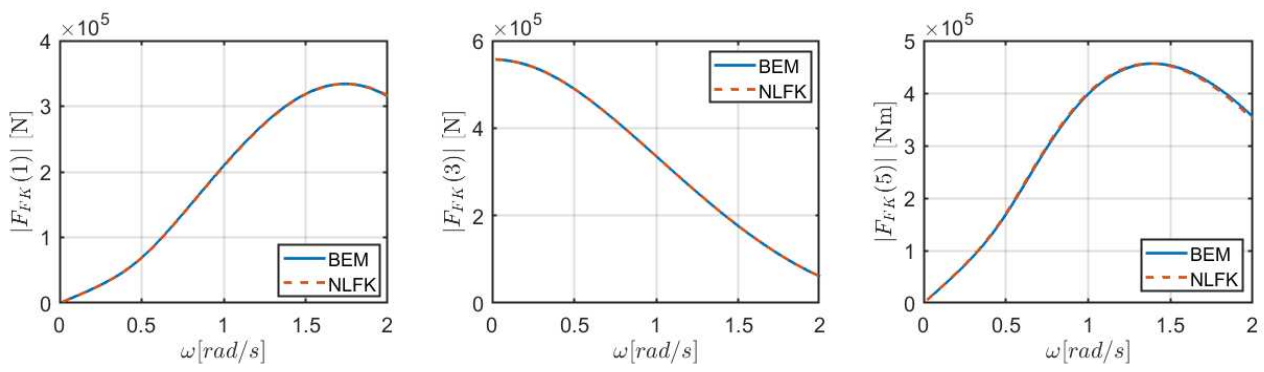

Figure 3: Comparison of the surge, heave, and pitch dynamic Froude-Krylov force per unit of wave amplitude, under linear conditions, using a linear BEM code and the NLFK approach.

$$
\begin{gathered}
\rho=\frac{R_{33}\left(C(3)-p_{1}-p_{0} C(1)\right)-q h(\vartheta)}{R_{13}^{2}+R_{23}^{2}-1+p_{0} R_{13} R_{33}+m h(\vartheta)} \\
h=\cos \vartheta\left(R_{11}\left(R_{13}+p_{0} R_{33}\right)+R_{21} R_{23}\right)+\sin \vartheta\left(R_{12}\left(R_{13}+p_{0} R_{33}\right)+R_{22} R_{23}\right)
\end{gathered}
$$

Figure 2 shows an example of a realistic wave energy converter, inspired by the Corpower device [18], represented in both the world frame and body frame after an arbitrary 6-DoF displacement. The buoy has a radius at the still water level of $4.2 \mathrm{~m}$ and a freeboard of $3 \mathrm{~m}$. A steep wave is also represented, with $5 \mathrm{~s}$ period and $2 \mathrm{~m}$ height (wavelength of $39 \mathrm{~m}$ ), in order to show the accuracy of the representation

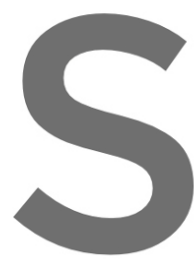
of the intersection, repres (5)). In fact, the horizont and the wavelength is rela significantly inaccurate, the liner

\subsection{Nonlinear Froude-Krylov force}
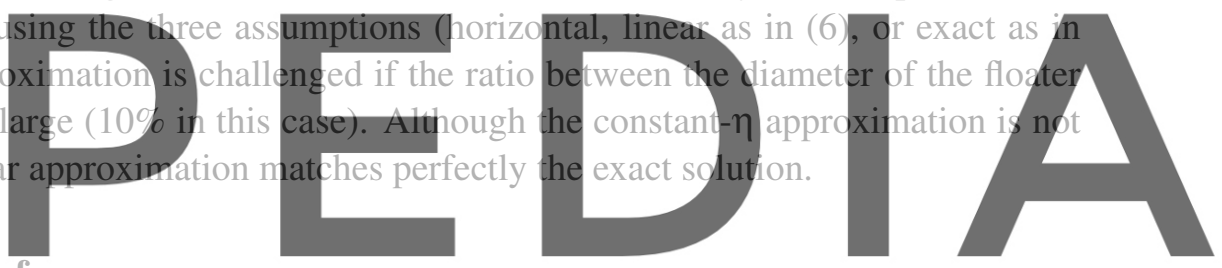

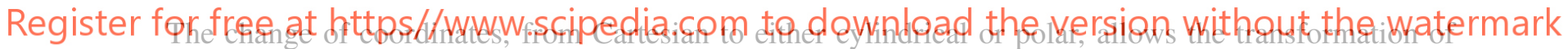

the 3D integral to 2D, as well as the representation of the normal vectors in parametric form. However,

note that the determinant of the Jacobian of the parametrization shouid be inciuded in the integral [1]. Such a mathematical framework is used to compute NLKF forces in 6-DoFs, which are the result of the gravity force and the integral of the undisturbed pressure field (static and dynamic) over the instantaneous wetted surface. Since it is convenient to write the equation of motion in the body-fixed frame (in order to have a constant inertial matrix), the NLFK forces are computed in the BF too. Moreover, it is both simpler and computationally more efficient to map the pressure field into the body frame, by means of the $4 \mathrm{D}$ rotation matrix $\mathrm{R}$, shown in (3). In order to validate the modelling approach and to verify the correctness of implementation, NLFK forces are computed in linear conditions and compared with linear solutions from boundary element method (BEM) software. Linear conditions are ensured by forcing zero displacement of the floater, and considering waves of $1 \mathrm{~mm}$ amplitude. The successful validation is shown in Figure 3. 


\section{Numerical integration algorithm and tolerances}

Since the computation time is the main perk of the proposed method, it is crucial to optimize the numerical integration algorithm and assess the impact of the chosen tolerances on the resulting error and computational burden. In the toolbox, the Matlab built-in function integral2 is used [19]. However, in this paper, a modified version of integral 2 is used, made more specific to the application case of the NLFK force calculation. Such a modification provides a reduction of the computational time between $25 \%$ and $40 \%$. However, due to ongoing development, this version is not yet implemented in the toolbox.

Generally, the domain of integration is a generalized rectangle, with $\vartheta \in[0,2 \pi]$ and $\rho \in\left[\rho_{1}(\vartheta), \rho_{2}(\vartheta)\right]$. The algorithm applies a mapping function in order to transform the domain into a proper rectangle, defined by $\alpha \in[0, \pi]$ and $\beta \in[0, \pi]$. Note that, due to the transformation of coordinates, the determinant of the Jacobian of the mapping function must be included in the integrand. The domain is then divided into 4 sub-rectangles, each one individually refined until a desired accuracy is obtained. The $2 \mathrm{D}$ integration is obtained through two linear quadrature formulae in cascade. A pair of a 3-point Kronrod and 7-point Gaussian formulae is used, realizing degrees of precision of 5 and 11, respectively [19]. Each one of these formulae simply applies a series of weights to the same function evaluations on a gridded selection of points on the domain, but achieve different degrees of accuracy. Consequently, both the estimate of the integral and the related error are obtained with just one set of function evaluations. Since the most expensive part of the integration is the integrand evaluation at each point of the domain, minimizing the computational time coincides with reducing the number of evaluations.
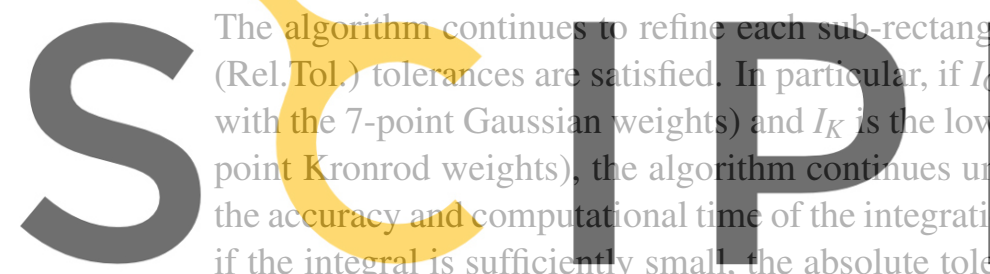

if the integral is sufficiently small, the absolute tol
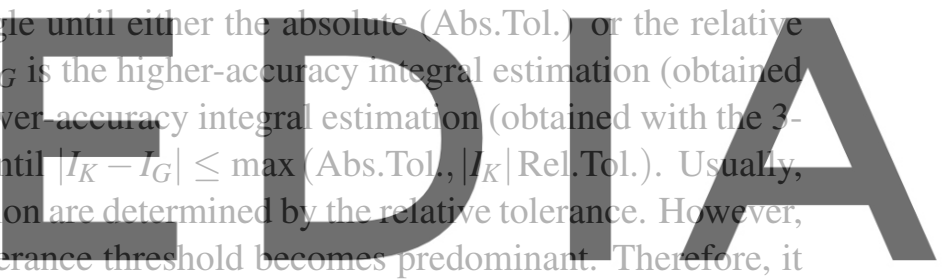

is important to investigate the importance of such tolerances in the specific case of NLFK forces.

Register for free at https/AwwWyscipedia, fom to downdoad the version without the watermark

zero-mean planar (surge, heave, and pitch) hydrodynamic excitation (diffraction and dynamic FK forces)

on axisymmetric devices. However, NLFK forces also comprises of the static FK component, which has zero mean in surge and pitch, but has positive mean in heave, equal to the static pressure integral over the wetted surface. This can be seen in the two top graphs in Figure 4, showing a time trace over a period of the heave NLFK force (top-left) and pitch NLFK force (top-right) for a representative regular wave of $2 \mathrm{~m}$ height and 1 $\mathrm{rad} / \mathrm{s}$ frequency, impacting the Corpower-like device, kept fixed at the rest position.

Forces in Figure 4 are computed for all combinations of 3 relative tolerances $\left(10^{-1}, 10^{-2}, 10^{-3}\right)$ and 9 logarithmically-equally spaced absolute tolerances, between $10^{-2}$ and $10^{-2}$. However, no difference can be appreciated, showing that the accuracy of the integration is significantly insensitive to such a range of tolerances (further quantification of the error depending on the accuracy is provided in Figures 5 and 6 ). Figure 4 also shows the computational time $\left(t_{\text {comp }}\right)$ for the evaluation of the individual NLFK force component at each of the 200 time instants the wave period is divided into. In order to average out stochastic variability of such small computational times, each $t_{\text {comp }}$ is computed as the average of 100 measures. The computational time for heave is about $1.5 \mathrm{~ms}$ and is insensitive to both tolerances and 


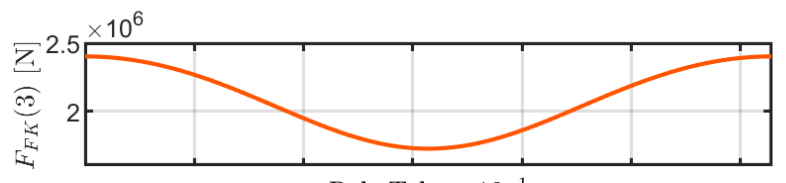

Rel. Tol. $=10^{-1}$
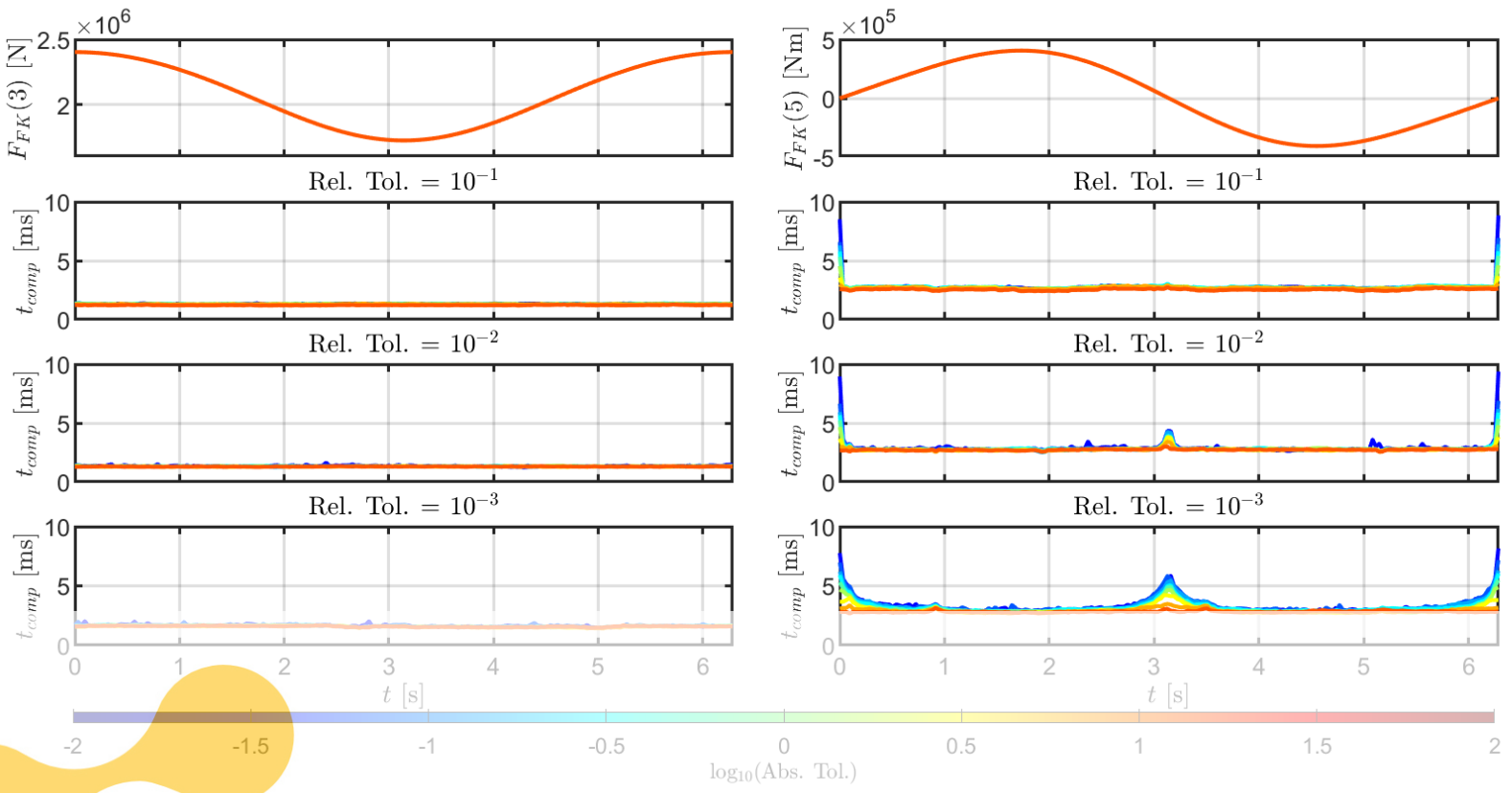

$\log _{10}($ Abs. Tol. $)$

Figure 4: On the top left and right, respectively, the nonlinear Froude-Krylov forces in heave and pitch for a Corpower-like device, fixed in the rest position, for a wave with $1 \mathrm{rad} / \mathrm{s}$ frequency and $2 \mathrm{~m}$ height. Below, the computational time for each time instant, each one computed as the average of 100 samples, are shown for three

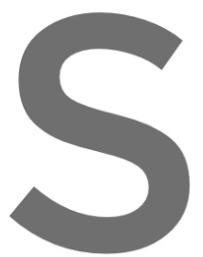

relative tolerances (from second to fourth rows) and 9 a
to the value of the integral, which is always of th
contrary, $t_{\text {comp }}$ in pitch is about constant only in re
about $2.8 \mathrm{~ms}$. Nevertheless, as the relative tolerance
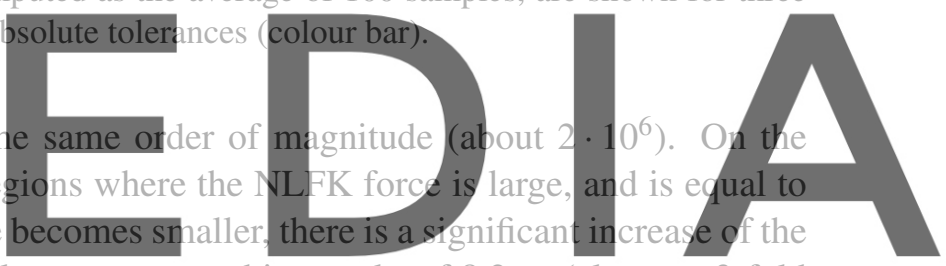

computational time in regions where the force is almost zero, reaching peaks of $8.2 \mathrm{~ms}$ (almost a 3-fold

Register for free at htheight/wnwidth of such an increase are inversely proportional to the absolute tolerance. Therefore, in order to reduce the computational burden, it is important to choose wisely the relative and

absolute tolerances, especially taking into account the values that the integral is expected to have.

A more throughout analysis of the sensitivity of the accuracy/computational time compromise to relative and absolute tolerances is necessary. Similarly to Figure 4, considering the device at rest, one wave period of a regular wave (frequency of $1 \mathrm{rad} / \mathrm{s}$ and height of $2 \mathrm{~m}$ ) is divided into 200 instants, in order to take the variability of the integrand into account. The nonlinear static and dynamic Froude-Krylov force $\left(F_{F K}\right)$ is computed in all DoFs, since nonlinear FK model may be used to investigate floaters under parametric resonance conditions [11]. One representative computational time to compute one time-instance of $F_{F K}$ for each $\operatorname{DoF}\left(t_{c o m p}(\mathrm{DoF})\right)$ is computed as the mean of all the time instants over a period. Note that such a measurement is averaged over 10 repetitions, in order to reduce stochastic variations.

A refined grid of absolute and relative tolerances is considered, taking all combinations of 33 logarithmically equally spaced tolerances between $10^{-4}$ and $10^{4}$. The integral using the smallest relative and absolute tolerances is taken as the accuracy benchmark. An estimate of the error is the root mean square error (rms) of the difference between the $F_{F K}$ at a given combination of tolerances and the benchmark. Figure 5 shows the error estimate (on the top) and the time step for a single force evaluation (on the 

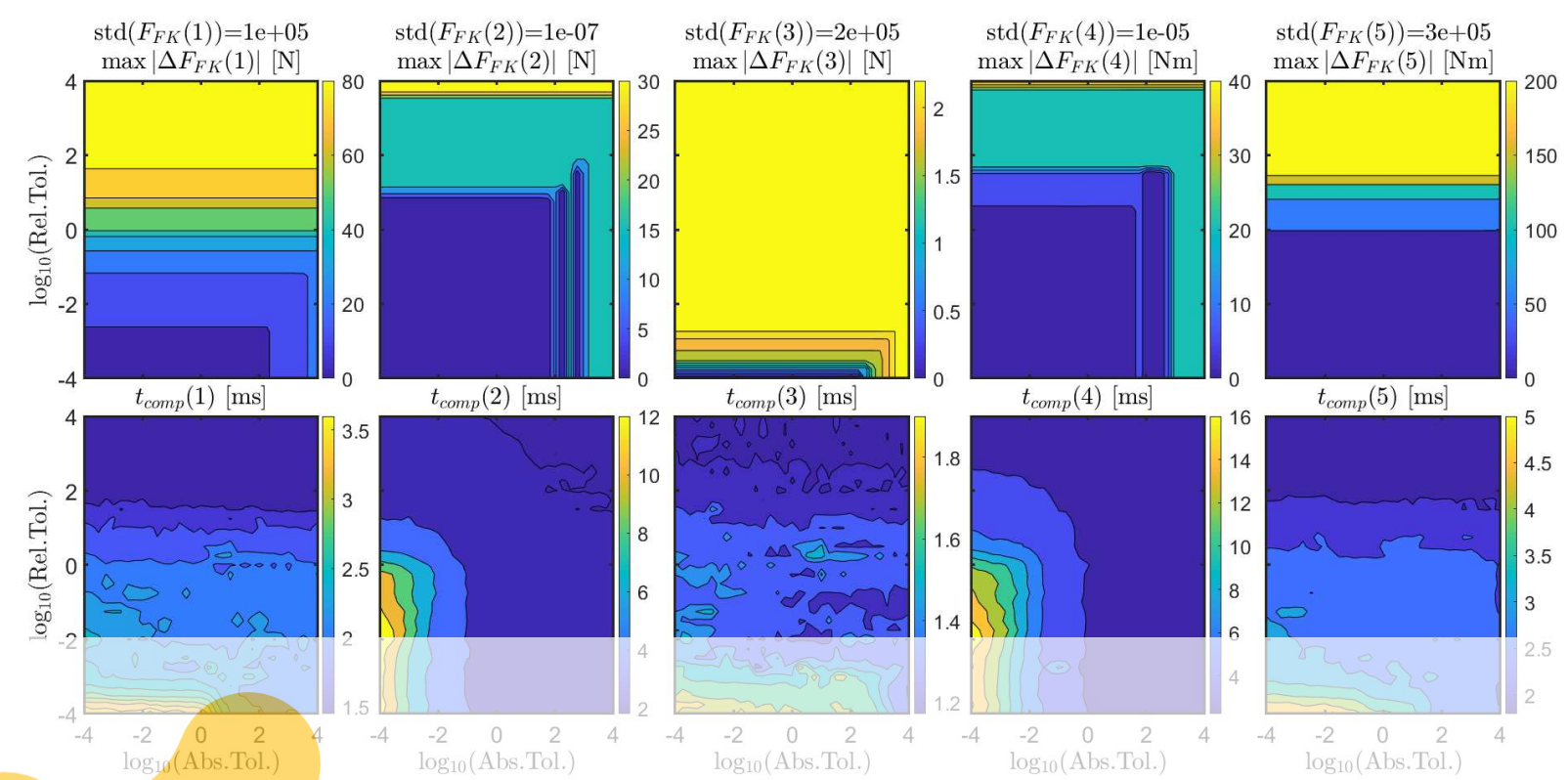

Figure 5: Absolute error (top line) and mean computational time for single evaluation (bottom line) for the calculation of nonlinear Froude-Krylov forces in surge, sway, heave, roll, and pitch (from left to right). Values are computed for a regular wave of $1 \mathrm{rad} / \mathrm{s}$ frequency and $2 \mathrm{~m}$ height, for a Corpower-like device fixed at the rest position.

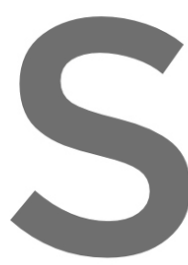

bottom) for 5 DoFs (surge,
force component is reporte
of the absolute error estim
value and several orders of
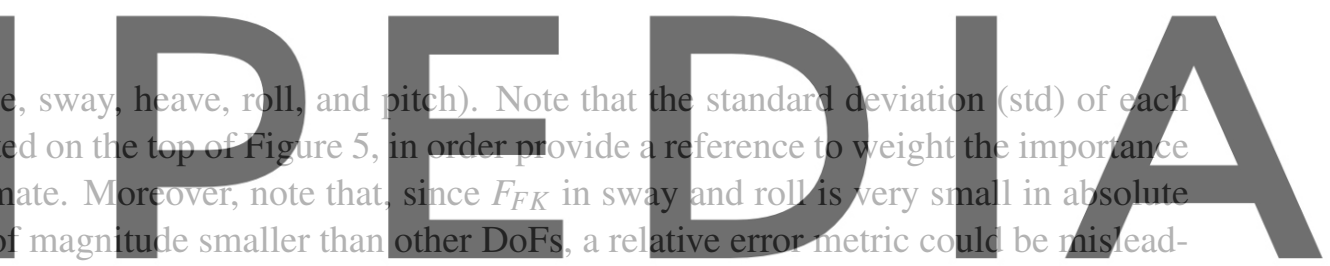

ing. On the other hand, the absolute error is more meaningful because it can be directly compared with

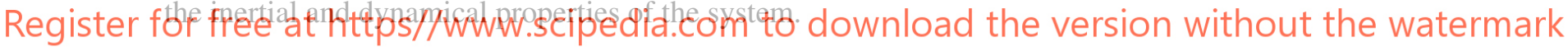

Figure 5 shows that the error in heave is negligible and significantly insensitive to the choice of tolerances; likewise the computational time shows small variations, from $1.2 \mathrm{~ms}$ to $1.9 \mathrm{~ms}$ per evaluation. Such an insensitivity is due to the non-zero mean of the heave force, as already shown in Figure 4. Moreover, it suggests that first approximations of the integration algorithm have already high accuracy, and few refinements are needed, probably thanks to the smooth nature of the integrand function.

Similarly to heave, also surge and pitch show a relatively small absolute error, compared to the standard deviation, apart from the upper bound of the relative tolerance range. Moreover, although insensitive to the absolute tolerance, there is a significant degradation of the performance as the relative tolerance increases. However, since surge and pitch FK forces have zero mean, the error increases when the integral is close to zero (if the relative tolerances are too large). Likewise, similarly to Figure 4, since additional iterations are needed to compute integrals close to zero, the computational time increases with decreasing relative tolerances, ranging from $1.5 \mathrm{~ms}$ to $5 \mathrm{~ms}$ per evaluation (larger variability then the zero-mean heave force). Note that, in general, surge and pitch require more time than heave.

Finally, note that the computational time required by the sway and roll DoF is significantly larger than 

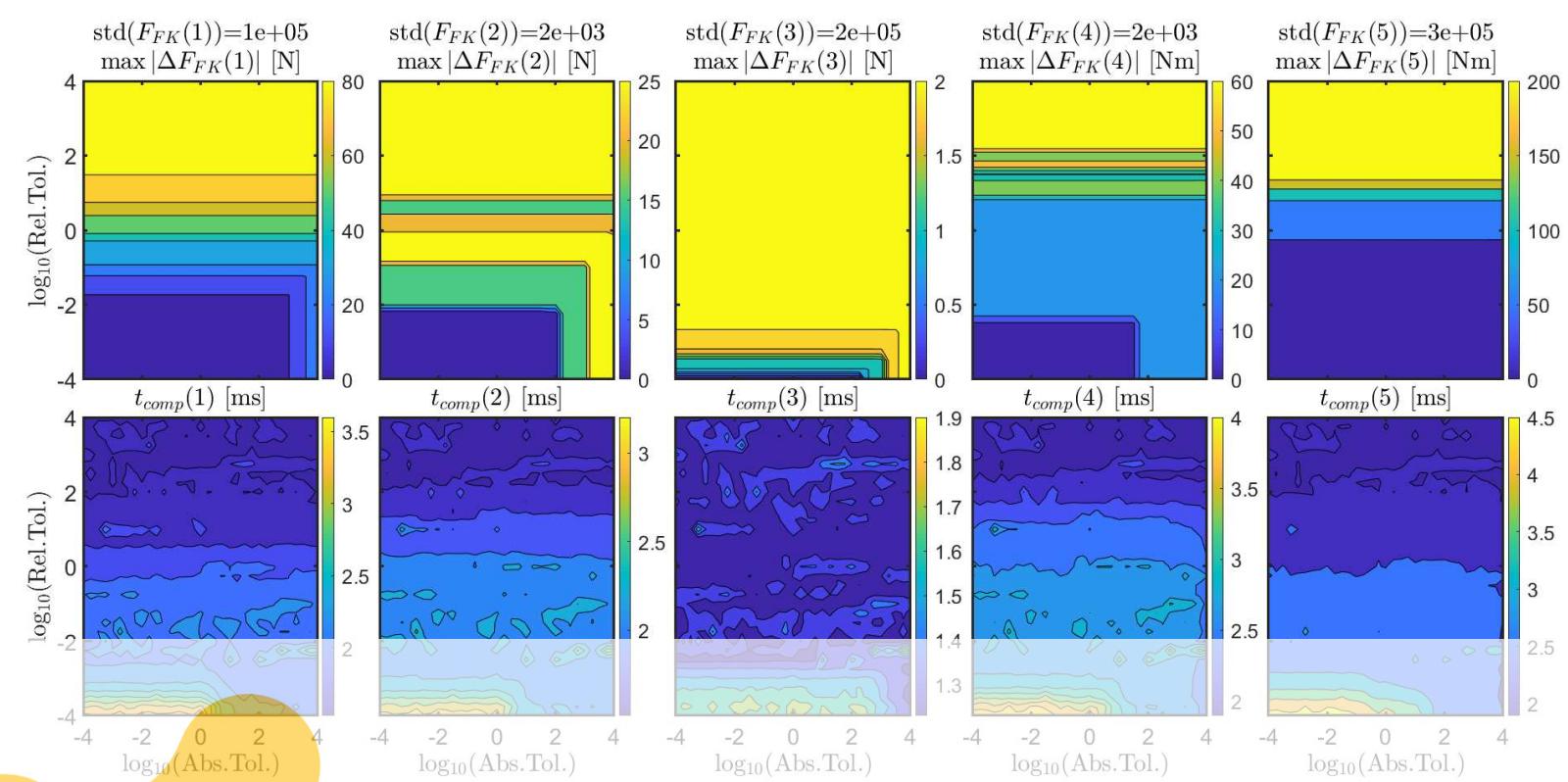

Figure 6: As in Figure 5, but with a perturbation of the device position of $0.01 \mathrm{~m}$ in the translational DoFs and $0.5^{\circ}$ in the rotational DoFs.

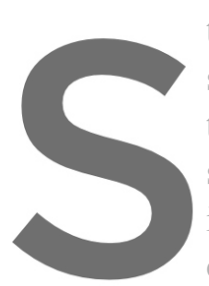

the other DoFs, and it

sway and roll FK forces a

the required accuracy. Hov

small forces and torques,

is never exactly at a positio

only more realistic, but
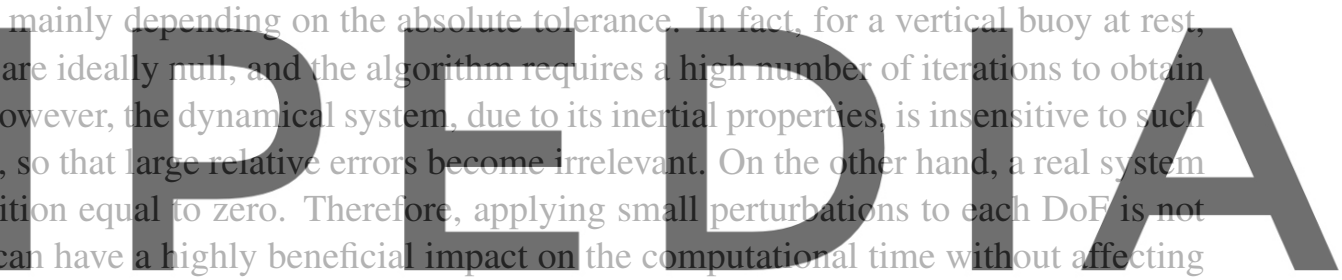

the dynamics of the system. In order to quantify this aspect, Figure 6 shows the same results of Figure

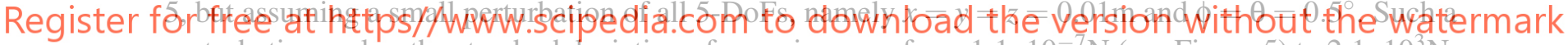
perturbation makes the standard deviation of sway increase from $1.1 \cdot 10^{-7} \mathrm{~N}$ (see Figure 5) to $2.1 \cdot 10^{3} \mathrm{~N}$, which is still 2 orders of magnitude smaller than the surge force. Similarly, the small perturbation makes the standard deviation of roll increase from $1.2 \cdot 10^{-5} \mathrm{Nm}$ (see Figure 5) to $2.5 \cdot 10^{3} \mathrm{Nm}$, which is still 2 orders of magnitude smaller than the pitch torque. However, the computational time of sway and roll undergo a multi-fold decrease, from a maximum of $16 \mathrm{~ms}$ in Figure 5 to a maximum of $4 \mathrm{~ms}$ in Figure 6. Moreover, the trends of the computational time maps of sway and roll become similar to the trends of sway and roll.

\section{Conclusions and guidelines}

This paper presents a mathematical framework for computationally efficient calculation of surface integrals over a time-varying surface of an axisymmetric body. The specific application case herein discussed is the calculation of nonlinear Froude-Krylov forces, for which there exist an open-source Matlab demonstration toolbox [1]. This paper details the strategy to compute the intersection between the floater and the free surface elevation. The numerical integration algorithm is also investigated, highlighting the im- 
pact of the integrand characteristics on the computational time and accuracy. Furthermore, a sensitivity to the relative and absolute tolerances is performed. In general, it is suggested to take into account both the expected values of each computed force, as well as the dynamical properties of the system to define the absolute tolerance, since floaters usually behave as low-pass filters. A reasonable starting guess is to use an absolute tolerance about 6 orders of magnitude lower than the inertia and a relative tolerance of about $10^{-2}$. However, it is highly suggested to perform further sensitivity analysis, since such tolerances depend on both the system under analysis and the objective pursued by the numerical model. Finally, in case of a multi-DoF simulation, it is suggested to avoid initial conditions of exactly zero, which significantly degrade the computational performance. Conversely, for virtually no change in the dynamic response of the system, it is suggested to apply a small perturbation to the initial condition.

Acknowledgement This research has received funding from the European Research Council (ERC) under the European Unions Horizon 2020 research and innovation program under Grant No. 832140.

\section{REFERENCES}

[1] G. Giorgi, Nonlinear Froude-Krylov Matlab demonstration toolbox (2019). doi:10.5281/zenodo.3544848.

[2] L. Genuardi, G. Bracco, S. A. Sirigu, M. Bonfanti, B. Paduano, P. Dafnakis, G. Mattiazzo, An application of model predictive control logic to inertial sea wave energy converter, in: Mechanisms and Machine Science, Vol. 73, Springer Netherlands, 2019, pp. 3561-3571. doi:10.1007/978-3030-20131-9_351.

[3] S. A. Sirigu, G. Bracco, M. Bonfanti, P. Dafnakis, G. Mattiazzo, On-board sea state estimation method validation based on measured floater motion, IFAC-PapersOnLine 51 (29) (2018) 68-73. doi:10.1016/J.IFACOL.2018.09.471.

[4] F. Wendt, K. Nielsen, Y.-h. Yu, H. Bingham, C. Eskilsson, B. Kramer, A. Babarit, T. Bunnik, R. Costello, S. Crowley, G. Giorgi, S. Giorgi, S. Girardin, D. Greaves, Ocean Energy Systems Wave Energy Modeling Task : Modeling, Verification, and Validation of Wave Energy Converters, Journal of Marine Science and Engineering 7 (379) (2019) 1-22. doi:10.3390/jmse7110379.

[5] G. Giorgi, J. V. Ringwood, Analytical Formulation of Nonlinear Froude-Krylov Forces for SurgingHeaving-Pitching Point Absorbers, in: ASME 2018 37th International Conference on Ocean, Offshore and Arctic Engineering, Madrid, 2018.

[6] G. Giorgi, J. V. Ringwood, Articulating Parametric Nonlinearities in Computationally Efficient Hydrodynamic Models, in: Proceedings of the 11th IFAC Conferecne on Control Applications in Marine Systems, Robotics, and Vehicles, Opatija, 2018.

[7] M. Lawson, Y.-H. Yu, A. Nelessen, K. Ruehl, C. Michelen, Implementing Nonlinear Buoyancy and Excitation Forces in the WEC-Sim Wave Energy Converter Modeling Tool, in: ASME 2014 33rd International Conference on Ocean, Offshore and Arctic Engineering,San Francisco, CA, American Society of Mechanical Engineers, 2014.

[8] H. Wang, A. Somayajula, J. Falzarano, Z. Xie, Development of a Blended Time-Domain Program for Predicting the Motions of a Wave Energy Structure, Journal of Marine Science and Engineering 
8 (1) (2019) 1. doi:10.3390/jmse8010001.

[9] G. Giorgi, J. V. Ringwood, Analytical representation of nonlinear Froude-Krylov forces for 3DoF point absorbing wave energy devices, Ocean Engineering 164 (2018) (2018) 749-759. doi:10.1016/j.oceaneng.2018.07.020.

[10] G. Giorgi, J. V. Ringwood, Parametric motion detection for an oscillating water column spar buoy., in: Proceedings of the 3rd International Conference on Renewable Energies Offshore RENEW, Lisbon, 2018.

[11] G. Giorgi, R. P. F. Gomes, G. Bracco, G. Mattiazzo, The Effect of Mooring Line Parameters in Inducing Parametric Resonance on the Spar-Buoy Oscillating Water Column Wave Energy Converter, Journal of Marine Science and Engineering 8 (1) (2020) 29. doi:10.3390/JMSE8010029.

[12] K. R. Tarrant, C. Meskell, Investigation on parametrically excited motions of point absorbers in regular waves, Ocean Engineering 111 (2016) 67-81. doi:10.1016/j.oceaneng.2015.10.041.

[13] J.-C. Gilloteaux, A. Babarit, G. Ducrozet, M. Durand, A. H. Clément, A non-linear potential model to predict large-amplitudes-motions: Application to the searev wave energy converter, Proceedings of the International Conference on Offshore Mechanics and Arctic Engineering - OMAE 4 (2007) 934-940. doi:10.1115/OMAE2007-29308.

[14] G. Bracco, M. Casassa, E. Giorcelli, G. Giorgi, M. Martini, G. Mattiazzo, B. Passione, M. Raffero, G. Vissio, Application of sub-optimal control techniques to a gyroscopic Wave Energy Converter, in: Renewable Energies Offshore, 2014, pp. 265-269.

[15] S. A. Sirigu, M. Bonfanti, B. Passione, E. Begovic, C. Bertorello, P. Dafnakis, G. Bracco, E. Giorcelli, G. Mattiazzo, Experimental investigation of the hydrodynamic performance of the ISWEC 1:20 scaled device, in: NAV International Conference on Ship and Shipping Research, no. 221499, 2018, pp. 551-560. doi:10.3233/978-1-61499-870-9-551.

[16] T. I. Fossen, Handbook of marine craft hydrodynamics and motion control, John Wiley and Sons, 2011.

[17] G. Giorgi, CCP-WSI Blind Test Series 3: A Nonlinear Froude-Krylov Modelling Approach, in: Y. S. Chung J.S., Akselsen O.M., Jin H., Kawai H., Lee Y., Matskevitch D., Ho Van S., Wan D., Wang A.M. (Ed.), Proceedings of the 29th International Ocean and Polar Engineering Conference, Vol. 3, International Society of Offshore and Polar Engineers, Honolulu, Hawaii, USA, 2019, pp. 3073-3080. doi:10.17736/ijope.2020.jc777.

[18] J. H. Todalshaug, G. S. Asgeirsson, E. Hjálmarsson, J. Maillet, P. Möller, P. Pires, M. Guérinel, M. F. Lopes, Tank testing of an inherently phase-controlled wave energy converter, International Journal of Marine Energy 15 (2016) 68-84.

[19] L. F. Shampine, Matlab program for quadrature in 2D, Applied Mathematics and Computation 202 (1) (2008) 266-274. doi:10.1016/j.amc.2008.02.012. 